\title{
AMICABLE PAIRS AND ALIQUOT CYCLES FOR ELLIPTIC CURVES OVER NUMBER FIELDS
}

\author{
JIM BROWN, DAVID HERAS, KEVIN JAMES, \\ RODNEY KEATON AND ANDREW QIAN
}

\begin{abstract}
Let $E / \mathbb{Q}$ be an elliptic curve. Silverman and Stange define primes $p$ and $q$ to be an elliptic, amicable pair if $\# E\left(\mathbb{F}_{p}\right)=q$ and $\# E\left(\mathbb{F}_{q}\right)=p$. More generally, they define the notion of aliquot cycles for elliptic curves. Here, we study the same notion in the case that the elliptic curve is defined over a number field $K$. We focus on proving the existence of an elliptic curve $E / K$ with aliquot cycle $\left(\mathfrak{p}_{1}, \ldots, \mathfrak{p}_{n}\right)$ where the $\mathfrak{p}_{i}$ are primes of $K$ satisfying mild conditions.
\end{abstract}

1. Introduction. The notion of amicable pairs of integers has been around since at least the Pythagoreans. Recall that a pair of positive integers $(m, n)$ is referred to as an amicable pair if the sum of the proper divisors of $m$ is equal to $n$ and the sum of the proper divisors of $n$ is $m$. The first such pair is given by $(220,284)$. There are many related notions to amicable numbers in elementary number theory; however, this paper is concerned with the notion of amicable pairs for elliptic curves as defined by Silverman and Stange [4].

Let $K$ be a number field, and let $E / K$ be an elliptic curve. We say that a pair of primes $(\mathfrak{p}, \mathfrak{q})$ of $\mathcal{O}_{K}$ form an amicable pair for $E$ if

$$
\begin{aligned}
& \# E\left(\mathbb{F}_{\mathfrak{p}}\right)=\mathrm{N} \mathfrak{q} \\
& \# E\left(\mathbb{F}_{\mathfrak{q}}\right)=\mathrm{N} \mathfrak{p},
\end{aligned}
$$

where we use $\mathrm{N}$ to denote the norm from $K$ to $\mathbb{Q}$ and where $\mathbb{F}_{\mathfrak{p}}$ denotes $\mathcal{O}_{K} / \mathfrak{p}$. More generally, one can define an elliptic aliquot cycle as a

2010 AMS Mathematics subject classification. Primary 11G05.

Keywords and phrases. Elliptic curves, amicable pairs, aliquot cycles.

All authors were partially supported by NSF grant No. DMS-1156734. The first author was also partially supported by NSA grant No. H98230-11-1-0137.

Received by the editors on October 30, 2014, and in revised form on January 17, 2015. 
collection of primes $\left(\mathfrak{p}_{1}, \ldots, \mathfrak{p}_{n}\right)$ satisfying

$$
\# E\left(\mathbb{F}_{\mathfrak{p}_{i}}\right)=\mathrm{N} \mathfrak{p}_{i+1} \quad \text { for } i=1, \ldots, n-1 \quad \text { and } \quad \# E\left(\mathbb{F}_{\mathfrak{p}_{n}}\right)=\mathrm{N} \mathfrak{p}_{1} \text {. }
$$

Aliquot cycles for elliptic curves defined over $\mathbb{Q}$ were studied extensively in [4]. Given an elliptic curve $E / \mathbb{Q}$, they provide asymptotics for the function $\mathcal{Q}_{E}(X)$ that counts the number of aliquot cycles $\left(p_{1}, \ldots, p_{n}\right)$ with $p_{1}=\min p_{i}$ and $p_{1} \leq X$. They also show that, for any positive integer $n$, there exists an elliptic curve $E / \mathbb{Q}$ that has an aliquot cycle of length $n$. This paper focuses on this existence result for elliptic curves over number fields.

We begin by showing that, if we allow primes of degree 1, we recover that, given any integer $n$ and any number field $K$, there is an elliptic curve $E / K$ that has an aliquot cycle of length $n$. The proof follows along the same lines as that given in [4], with the only added input being the distribution of primes of degree 1. However, if we restrict to the case where at least one of the primes is required to have degree at least 2, things are very different. This is to be expected as the density of primes of degree greater than 1 is much thinner than that of degree 1 primes. We note that any sequence of primes with a common norm forms an aliquot cycle (see the discussion immediately following Theorem 2.4), and thus, it is of interest to focus on elliptic aliquot cycles involving more than one norm.

We prove that the only possible such sequences of primes $\left(\mathfrak{p}_{1}, \ldots, \mathfrak{p}_{n}\right)$ with equal degree $f>1$ are for $n=f=2$ and $\mathfrak{p}_{1}\left|2, \mathfrak{p}_{2}\right| 3$. Then, we study the case of primes with unequal degrees possibly greater than 1 . This case is interesting in that it is possible to have such aliquot cycles. In fact, we give a criterion for a sequence of primes to be an elliptic aliquot cycle (see Theorem 2.4). We also provide an algorithm for constructing such an elliptic curve and give two explicit examples.

Finally, we conclude with a section describing some potential future research expanding these notions to hyperelliptic curves.

\section{Existence of aliquot cycles for elliptic curves over num-}

ber fields. First, we establish necessary conditions for a sequence $\mathfrak{p}_{1}, \mathfrak{p}_{2}, \ldots, \mathfrak{p}_{n}$ of primes of $\mathcal{O}_{K}$ to be an aliquot cycle for some elliptic curve $E / K$. Note that the Hasse bound tells us that, if our sequence is to be an elliptic aliquot cycle, then the norms of consecutive primes 
in our sequence must be close together. In fact, if $\mathfrak{p}$ and $\mathfrak{q}$ are primes appearing consecutively in the sequence (where we consider $\mathfrak{p}_{n}$ and $\mathfrak{p}_{1}$ as appearing consecutively), then the Hasse bound requires that

$$
|\mathrm{N} \mathfrak{p}+1-\mathrm{Nq}| \leq 2 \sqrt{\mathrm{Np}}
$$

We recall the following consequence of Deuring's theorem due to Schoof that further restricts which sequences of primes can be elliptic aliquot cycles. The statement given here is a special case of [3, Theorem 4.2], combined with Mihăilescu's theorem [2, Catalan's conjecture].

Theorem 2.1 (Deuring-Schoof). Suppose that $K$ is a number field with a ring of integers $\mathcal{O}_{K}$ and that $\mathfrak{p}$ and $\mathfrak{q}$ are primes of $\mathcal{O}_{K}$ lying above the rational primes $p$ and $q$, respectively. There is an elliptic curve $E / \mathbb{F}_{\mathfrak{p}}$ with $\# E\left(\mathbb{F}_{\mathfrak{p}}\right)=\mathrm{Nq}$ if and only if one of the following conditions holds.

(i) $(\mathrm{Np}, \mathrm{Nq}-1)=1$ and $|\mathrm{Np}+1-\mathrm{Nq}| \leq 2 \sqrt{\mathrm{Np}}$,

(ii) $\mathrm{Np}=p^{2 r}$ with $p \not \equiv 1(\bmod 3)$ and $N \mathfrak{q}=p^{2 r} \pm p^{r}+1$,

(iii) $\mathrm{Np}=3^{2 r+1}$ and $\mathrm{Nq}=3^{2 r+1} \pm 3^{r+1}+1$,

(iv) $\mathrm{Np}=2^{2 r+1}$ and $\mathrm{Nq}=2^{2 r+1} \pm 2^{r+1}+1$,

(v) $(\mathrm{N} \mathfrak{p}, \mathrm{N} \mathfrak{q})=\left(2^{r}-1,2^{r}\right),\left(\left(2^{r}-1\right)^{2}, 2^{2 r}\right)$, or $\left(2^{2 r},\left(2^{r}-1\right)^{2}\right)$, provided that $2^{r}-1$ is prime.

(vi) $(\mathrm{Np}, \mathrm{Nq})=(2,3),(4,9),(9,4),(8,9),(81,64),(64,81),(4,5)$, $(16,17),(256,257),(65536,65537),(16,25),(256,289),(65536$, $66049),\left(65536^{2}, 65537^{2}\right),(25,16),(289,256),(66049,65536)$ or $\left(65537^{2}, 65536^{2}\right)$.

(vii) $(\mathrm{Np}, \mathrm{Nq})=\left(2^{2^{k}}, 2^{2^{k}}+1\right),\left(\left(2^{2^{k}}+1\right)^{2}, 2^{2^{k+1}}\right)$, or $\left(2^{2^{k+1}},\left(2^{2^{k}}+1\right)^{2}\right)$, provided $2^{2^{k}}+1$ is prime.

Definition 2.2. A collection of primes $\mathfrak{p}_{1}, \ldots, \mathfrak{p}_{n}$ is a Deuring $n$-cycle if it has the property that each pair of primes appearing consecutively in the sequence (where $\left(\mathfrak{p}_{n}, \mathfrak{p}_{1}\right)$ are included as consecutive primes from the sequence) satisfies one of the conditions of Theorem 2.1.

Remark 2.3. Suppose that we are given a sequence of primes in some number field $K$, and we wish to know if it is a Deuring $n$-cycle. Let $\mathfrak{p}$ and $\mathfrak{q}$ be consecutive primes of the sequence lying above rational primes $p$ and $q$, respectively. 
(i) If $p, q \geq 5$, then only conditions (i) and (ii) need to be considered.

(ii) If $p, q \geq 3$, then only conditions (i), (ii) and (iii) need to be considered.

(iii) Condition (vii) relies on the existence of Fermat primes and is likely to be superfluous since we have included the contributions of this form from the known Fermat primes in condition (vi).

This gives the following criterion for a sequence of primes to be an elliptic aliquot cycle.

Theorem 2.4. Suppose that $K$ is a number field with a ring of integers $\mathcal{O}_{K}$. A sequence $\mathfrak{p}_{1}, \ldots, \mathfrak{p}_{n}$ of primes of $\mathcal{O}_{K}$ is an aliquot cycle for some elliptic curve $E / K$ if and only if it is a Deuring $n$-cycle. Furthermore, if the sequence is a Deuring $n$-cycle, then it is an aliquot cycle for infinitely many elliptic curves $E / K$.

Proof. Suppose $\mathfrak{p}_{1}, \ldots, \mathfrak{p}_{n}$ is an aliquot cycle for some elliptic curve $E / K$. Then, it follows immediately from Theorem 2.1 that $\mathfrak{p}_{1}, \ldots, \mathfrak{p}_{n}$ is a Deuring $n$-cycle.

Now, suppose that $\mathfrak{p}_{1}, \ldots, \mathfrak{p}_{n}$ is a Deuring $n$-cycle. Under our hypothesis, Theorem 2.1 guarantees the existence of a curve $E_{i} / \mathbb{F}_{\mathfrak{p}_{i}}$ with $\# E\left(\mathbb{F}_{\mathfrak{p}_{i}}\right)=\mathrm{Np}_{i+1}$ and the existence of a curve $E_{n} / \mathbb{F}_{\mathfrak{p}_{n}}$ with $\# E\left(\mathbb{F}_{\mathfrak{p}_{n}}\right)=\mathrm{Np}_{1}$, for each $1 \leq i \leq n-1$. Thus, we can use the Chinese remainder theorem to construct an elliptic curve $E / K$ whose coefficients are congruent to those of $E_{i}$ modulo $\mathfrak{p}_{i}$ for $1 \leq i \leq n$, and the sequence $\mathfrak{p}_{1}, \ldots, \mathfrak{p}_{n}$ will be an aliquot cycle for any curve which has coefficients congruent modulo $\mathfrak{p}_{1} \cdot \ldots \cdot \mathfrak{p}_{n}$ to those of $E$.

Now, we are in a position to prove the existence of aliquot sequences in any number field $K$. First, we note that, if $\mathfrak{p}_{1}, \ldots, \mathfrak{p}_{n}$ is a sequence of primes of $\mathcal{O}_{K}$ with a common norm, then Theorem 2.1 (i) is met, and thus, there are infinitely many elliptic curves $E / K$ for which the sequence is aliquot. Henceforth, we will focus our search on elliptic aliquot cycles involving more than one norm. Note that, for such an aliquot cycle, we can extend the cycle by adding primes of a common norm. We should also note that primes of a common norm may appear nonconsecutively in an aliquot cycle. This will give us more freedom in satisfying the conditions of Theorem 2.4. 
The next theorem essentially follows from the arguments given in [4]. We include it with proof for the sake of completeness.

Theorem 2.5. Given a number field $K$ and a natural number $n \in \mathbb{N}$, there are infinitely many length $n$ sequences of primes $\mathfrak{p}_{1}, \mathfrak{p}_{2}, \ldots, \mathfrak{p}_{n} \subseteq$ $\mathcal{O}_{K}$ with pairwise distinct norms, and, for each such sequence infinitely many elliptic curves $E$ satisfying $E\left(\mathbb{F}_{\mathfrak{p}_{i}}\right)=\mathrm{Np}_{i+1}$ for $1 \leq i \leq n-1$ and $E\left(\mathbb{F}_{\mathfrak{p}_{n}}\right)=\mathrm{N} \mathfrak{p}_{1}$.

Proof. Let $K$ be any number field, and let $n \in \mathbb{N}$. In light of Theorem 2.4, it will be sufficient to show that there are infinitely many Deuring $n$-cycles with pairwise distinct norms. This is guaranteed by the Chebotarev density theorem. To see this, note that if, for some $n$, there were no sequence of degree 1 prime ideals with pairwise distinct norms $\mathfrak{p}_{1}, \mathfrak{p}_{2}, \ldots, \mathfrak{p}_{n} \subseteq \mathcal{O}_{K}$ satisfying the condition

$$
\mathrm{Np}_{1}<\mathrm{Np}_{2}<\cdots<\mathrm{Np}_{n}<\mathrm{Np}_{1}+2 \sqrt{\mathrm{Np}_{1}},
$$

then the rational primes up to any bound $X$ which split completely in $\mathcal{O}_{K}$ would be less numerous than $n$ times the number of squares up to $X$, which would violate the Chebotarev theorem. Now, note that, if condition (2.2) is satisfied for a sequence of degree 1 primes with pairwise distinct norms, then Theorem 2.1 (i) is satisfied, and the sequence is indeed a Deuring $n$-cycle. Thus, we are guaranteed infinitely many length $n$ sequences of prime ideals $\mathfrak{p}_{1}, \mathfrak{p}_{2}, \ldots, \mathfrak{p}_{n} \subseteq \mathcal{O}_{K}$ satisfying the hypotheses of Theorem 2.4, and Theorem 2.5 follows.

We note that the aliquot cycles given by this technique may all be made up of primes of degree 1 and thus are not much different than the sequences exhibited in [4]. Thus, it is interesting to search for elliptic curves $E / K$ which have aliquot cycles involving primes of degree greater than 1 .

3. Amicable pairs of primes of degree greater than 1 . In the previous section, we showed that the behavior of degree 1 primes over number fields mirrors that of the case over $\mathbb{Q}$, previously studied by Silverman and Stange [4]. In this section, we see that the situation is much different if we consider primes of degree greater than 1 . We begin with the following result. 
Theorem 3.1. Let $E / K$ be an elliptic curve. Let $\mathfrak{p}$ and $\mathfrak{q}$ be primes of the same degree $f>1$ but with different norms. Then, $(\mathfrak{p}, \mathfrak{q})$ forms an amicable pair if and only if $[K: \mathbb{Q}]$ is even and $(\mathrm{N} \mathfrak{p}, \mathrm{Nq})=(4,9)$.

Proof. Let $E / K$ be an elliptic curve. Let $\mathfrak{p}$ and $\mathfrak{q}$ be primes of degree $f \geq 2$ with differing norms. Suppose that they form an amicable pair. Let $p$ be the rational prime so that $\mathfrak{p} \mid p$, and let $q$ be the rational prime so that $\mathfrak{q} \mid q$. Without loss of generality, assume that $p<q$. From Hasse's bound, we have that

$$
\left|q^{f}-p^{f}-1\right|=\left|\# E\left(\mathbb{F}_{\mathfrak{p}}\right)-p^{f}-1\right| \leq 2 p^{f / 2} .
$$

Note that we are essentially measuring the distance between the prime powers $p^{f}$ and $q^{f}$. Now, it is a simple matter to show that, if $f \geq 2$, then they cannot be this close together unless $p=2, q=3$ and $f=2$.

First, suppose that $p=2, q=3$ and $f>2$. Then, we have

$$
\begin{aligned}
\left|3^{f}-2^{f}-1\right| & =\left|(2+1)^{f}-2^{f}-1\right| \\
& =\left|\sum_{j=0}^{f}\left(\begin{array}{l}
f \\
j
\end{array}\right) 2^{f-j}-2^{f}-1\right| \\
& \geq f 2^{f-1}>2 \cdot 2^{f / 2},
\end{aligned}
$$

where the last inequality follows from the fact that $f>2$. Thus, such a $(\mathfrak{p}, \mathfrak{q})$ cannot form an amicable pair, since it violates the Hasse bound (2.1).

Now, suppose that $q>p>2$. The value $\left|q^{f}-p^{f}-1\right|$ is minimized when $q=p+2$. Arguing as above, we have

$$
\left|(p+2)^{f}-p^{f}-1\right|>2 f p^{f-1}>2 p^{f / 2},
$$

where we have used that $f \geq 2$. This clearly violates the Hasse bound (2.1).

Thus, the only possibility for $(\mathfrak{p}, \mathfrak{q})$ to be amicable is if $(\mathrm{Np}, \mathrm{Nq})=$ $(4,9)$. Finally, if $(\mathrm{Np}, \mathrm{Nq})=(4,9)$, then Theorem 2.1 (vi) is satisfied, and thus, $(\mathfrak{p}, \mathfrak{q})$ is a Deuring 2-cycle. Theorem 3.1 now follows from Theorem 2.4 .

We have the following example of such a number field $K$. 
Example 3.2. Let $K=\mathbb{Q}(\sqrt{5})$. We have that 2 and 3 are both inert in this field. Set $\mathfrak{p}_{2}=2 \mathcal{O}_{K}$ and $\mathfrak{p}_{3}=3 \mathcal{O}_{K}$. Then, $\mathrm{Np}_{2}=2^{2}$ and $\mathrm{Np}_{3}=3^{2}$. We use Sage [5] to see that the elliptic curve $E_{2} / K: y^{2}+y=x^{3}$ satisfies $\# E_{2}\left(\mathbb{F}_{\mathfrak{p}_{2}}\right)=3^{2}$ and $E_{3} / K: y^{2}=x^{3}+2 \sqrt{5} x$ satisfies $\# E_{3}\left(\mathbb{F}_{\mathfrak{p}_{3}}\right)=2^{2}$. The Chinese remainder theorem is used to combine these curves to form

$$
\frac{E}{K}: y^{2}+3 y=x^{3}+2 \sqrt{5} x .
$$

This curve has $\left(\mathfrak{p}_{2}, \mathfrak{p}_{3}\right)$ as an amicable pair.

Our next step is to consider the case where $\mathfrak{p}$ and $\mathfrak{q}$ are primes of degrees $e$ and $f$, respectively, with $e \neq f$. This naturally leads to the question: are there any number fields $K$ that have Deuring $n$-cycles for $n>2$ ? In the next section, we address this question by giving a method for constructing such number fields given rational prime powers satisfying mild conditions. We also provide some specific examples.

4. Existence of number fields with Deuring cycles. In this section, we give a method for constructing examples of number fields $K$ that have Deuring cycles.

Definition 4.1. A sequence of rational prime powers $p_{1}^{f_{1}}, \ldots, p_{n}^{f_{n}}$ is a potential Deuring $n$-cycle provided that, if we could find a number field $K$ and a sequence of primes $\mathfrak{p}_{1}, \ldots, \mathfrak{p}_{n} \subseteq \mathcal{O}_{K}$ with $\mathrm{Np}_{i}=p_{i}^{f_{i}}$ for $1 \leq i \leq n$, then this sequence would be a Deuring $n$-cycle.

We will show that, given a potential Deuring $n$-cycle where prime powers are allowed to be repeated a limited number of times, there is a number field $K$ and a Deuring $n$-cycle of primes in $\mathcal{O}_{K}$ with norms given by the prime powers in the given sequence. We construct two examples. For the first, we construct a specific number field $K$, a Deuring 2-cycle $(\mathfrak{p}, \mathfrak{q})$ and an elliptic curve $E / K$ for which $(\mathfrak{p}, \mathfrak{q})$ forms an amicable pair. For the second, we give a specific number field $K$, a Deuring 10-cycle, and an elliptic curve $E / K$ for which the Deuring 10-cycle forms an aliquot cycle.

We will make use of the following well-known theorem. 
Theorem 4.2. Let $K=\mathbb{Q}(\alpha)$ where $\alpha$ is a root of an irreducible polynomial $f(x) \in \mathbb{Z}[x]$. Let $p$ be a prime with $p \nmid\left[\mathcal{O}_{K}: \mathbb{Z}[\alpha]\right]$. If $f(x)$ has irreducible factorization in $\mathbb{F}_{p}[x]$ given by $f(x)=g_{1}(x) \cdots g_{k}(x)$, then

$$
p \mathcal{O}_{K}=\prod_{i=1}^{k}\left\langle p, g_{i}(\alpha)\right\rangle .
$$

Using Theorem 4.2, we have the following method for constructing an appropriate number field.

Corollary 4.3. Let $p_{1}^{f_{1}}, \ldots, p_{n}^{f_{n}}$ be a potential Deuring $n$-cycle with the additional property that the number of occurrences of any prime power $p^{f}$ does not exceed the number of monic irreducible polynomials of degree $f$ over $\mathbb{F}_{p}$. Then there exists a number field $K$ and primes $\mathfrak{p}_{1}, \ldots, \mathfrak{p}_{n} \subset \mathcal{O}_{K}$ such that $\mathrm{Np}_{i}=p_{i}^{f_{i}}$, i.e., $\left(\mathfrak{p}_{1}, \ldots, \mathfrak{p}_{n}\right)$ is a Deuring $n$-cycle for $K$.

Proof. Let $p_{1}^{f_{1}}, \ldots, p_{n}^{f_{n}}$ be a potential Deuring $n$-cycle with the additional property that the number of occurrences of any prime power $p^{f}$ in the sequence does not exceed the number of monic irreducible polynomials of degree $f$ over $\mathbb{F}_{p}$. Let us denote the distinct primes in the sequence as $q_{1}, \ldots, q_{m}$, and let us denote the not necessarily distinct powers of $q_{i}$ by $q_{i}^{f_{i, 1}}, \ldots, q_{i}^{f_{i, k_{i}}}$.

For each $1 \leq i \leq m$ and $1 \leq j \leq k_{i}$, let $h_{i, j}(x) \in \mathbb{F}_{q_{i}}[x]$ be an irreducible monic polynomial of degree $f_{i, j}$ chosen such that $h_{i, 1}, \ldots, h_{i, k_{i}}$ are distinct. Note that this can be done since we limit the number of occurrences of $q_{i}^{f_{i, j}}$ in our sequence to less than or equal to the number of monic irreducible polynomials in $\mathbb{F}_{q_{i}}[x]$ of degree $f_{i, j}$. Now, choose $D>1$ large enough such that, for each $1 \leq i \leq m$, we can choose a monic irreducible polynomial $g_{i} \in \mathbb{F}_{q_{i}}[x]$ of degree

$$
D-\sum_{j=1}^{k_{i}} f_{i, j},
$$

which is distinct from $h_{i, 1}, \ldots, h_{i, k_{i}}$.

Now, select any prime $r$ which does not divide any member of our sequence and a monic irreducible polynomial $k(x) \in \mathbb{F}_{r}[x]$ of 
degree $D$. Apply the Chinese remainder theorem to the coefficients of the polynomials

$$
g_{i}(x) \prod_{j=1}^{k_{i}} h_{i, j}(x), \quad 1 \leq i \leq m,
$$

and $k(x)$ to construct a polynomial $F(x) \in \mathbb{Z}[x]$ so that

$$
F(x) \equiv g_{i}(x) \prod_{j=1}^{k_{i}} h_{i, j}(x) \quad\left(\bmod q_{i}\right)
$$

for each $1 \leq i \leq m$ and $F(x) \equiv k(x)(\bmod r)$. Since $k(x)$ is irreducible modulo $r$, we must have that $F(x)$ is irreducible in $\mathbb{Q}[x]$. Let $\alpha \in \overline{\mathbb{Q}}$ be a root of $F(x)$ and set $K=\mathbb{Q}(\alpha)$.

It only remains to show that $q_{i} \nmid\left[\mathcal{O}_{K}: \mathbb{Z}[\alpha]\right]$ by applying Theorem 4.2. Recall that $\left[\mathcal{O}_{K}: \mathbb{Z}[\alpha]\right] \mid \operatorname{disc}(F)$, so it is enough to show that $q_{i} \nmid \operatorname{disc}(F)$ for $1 \leq i \leq m$. Since, for each $1 \leq i \leq m, \mathbb{F}_{q_{i}}$ is perfect, and since the irreducible polynomials $g_{i}, h_{i, 1}, \ldots, h_{i, k_{i}}$, are distinct, it follows that

$$
g_{i}(x) \prod_{j=1}^{k_{i}} h_{i, j}(x)
$$

has $D$ distinct roots in $\overline{\mathbb{F}}_{q_{i}}$, say $\alpha_{i, 1}, \ldots, \alpha_{i, D}$. Furthermore, since the discriminant of a polynomial can be expressed in terms of its roots, we obtain, by our construction of $F$, that

$$
\begin{aligned}
\operatorname{disc}(F) & \equiv \operatorname{disc}\left(g_{i}(x) \prod_{j=1}^{k_{i}} h_{i, j}(x)\right) \quad\left(\bmod q_{i}\right) \\
& =\prod_{i<j}\left(\alpha_{i}-\alpha_{j}\right)^{2} \not \equiv 0 \quad\left(\bmod q_{i}\right) .
\end{aligned}
$$

Thus, we have the result.

Once a potential Deuring $n$-cycle of prime powers satisfying the hypothesis of Corollary 4.3 has been found, it is fairly easy to construct a suitable field $K$ using the method of our proof. We provide the following explicit examples. 
Example 4.4. Let $p_{1}=13, f_{1}=3, p_{2}=47$ and $f_{2}=2$. Observe that

$$
\begin{aligned}
\left|47^{2}-13^{3}-1\right| & =11 \leq 2 \cdot 13^{3 / 2} \\
\left|13^{3}-47^{2}-1\right| & =13 \leq 2 \cdot 47, \\
\left(13^{2}, 47^{2}-1\right) & =1
\end{aligned}
$$

and

$$
\left(47^{2}, 13^{2}-1\right)=1
$$

so this is a potential Deuring 2-cycle. Let

$$
f(x)=x^{3}-52 x+329 \text { and } K=\mathbb{Q}(\alpha)
$$

where $\alpha$ is a root of $f(x)$. Let

$$
\mathfrak{p}_{13}=\left\langle 13, \alpha^{3}+4\right\rangle \quad \text { and } \quad \mathfrak{p}_{47}=\left\langle 47, \alpha^{2}-5\right\rangle .
$$

We use Sage to find that

$$
\frac{E_{13}}{K}: y^{2}=x^{3}+\left(10 \alpha^{2}+9 \alpha+12\right) x+\left(9 \alpha^{2}+11 \alpha+9\right)
$$

satisfies $\# E_{13}\left(\mathbb{F}_{\mathfrak{p}_{13}}\right)=47^{2}$ and

$$
\frac{E_{47}}{K}: y^{2}=x^{3}+(46 \alpha+11) x+(20 \alpha+37)
$$

satisfies $\# E_{47}\left(\mathbb{F}_{\mathfrak{p}_{47}}\right)=13^{3}$. We then apply the Chinese remainder theorem to these curves to find $E / K$, given by

$$
\frac{E}{K}: y^{2}=x^{3}+\left(517 \alpha^{2}+516 \alpha+246\right) x+\left(282 \alpha^{2}+349 \alpha+178\right)
$$

is an elliptic curve that satisfies $\# E\left(\mathbb{F}_{\mathfrak{p}_{1}}\right)=\mathrm{N} \mathfrak{p}_{2}$ and $\# E\left(\mathbb{F}_{\mathfrak{p}_{2}}\right)=\mathrm{N} \mathfrak{p}_{1}$, as desired.

We also provide an example of an aliquot cycle of greater length.

Example 4.5. Consider the cycle given by $\left(2^{2}, 3,5,7,11,13,11,7,5,3\right)$. One can easily verify that this is a potential Deuring cycle. Following the algorithm in the proof of Corollary 4.3, one sees that the number field given by $\mathbb{Q}(\alpha)$ with $\alpha$ a root of $f(x)=x^{2}+x+195195$ realizes 
$\left(2^{2}, 3,5,7,11,13,11,7,5,3\right)$ as a Deuring 10-cycle. MAGMA [1] can then be used to show $E / K$, given by

$$
\frac{E}{K}: y^{2}+\alpha y=x^{3}+100010 x^{2}+(98 \alpha+12552) x-(716 \alpha+10004)
$$

has $\left(2^{2}, 3,5,7,11,13,11,7,5,3\right)$ as an aliquot cycle.

Remark 4.6. We recall that Legendre conjectured that there is always a prime between consecutive integer squares, and computational evidence seems to suggest more. In fact, it seems reasonable to conjecture that there are as many as $\sqrt{n}$ primes between $n^{2}$ and $(n+1)^{2}$. Based on this, it seems reasonable that there may always be a prime between

$$
x=(\sqrt{x})^{2} \text { and } \quad x+2 \sqrt{x}=(\sqrt{x}+1)^{2}-1 .
$$

If so, one could construct an arbitrarily long sequence of prime powers starting at any prime power which could be realized as the norm sequence of a Deuring cycle of primes of some number field $K$, as constructed above. To find such a sequence of prime powers, one simply continues to select prime powers, each within twice the square root of the previous one, until satisfied. One then repeats the sequence in reverse, being careful not to reuse 4 , if it was used before, until again reaching the beginning.

5. Future directions of research: hyperelliptic curves. If one wishes to generalize the notion of amicable pairs for elliptic curves, a very natural object to look at is a hyperelliptic curve.

Definition 5.1. Let $K$ be a field. A hyperelliptic curve $C / K$ of genus $g \geq 1$ is a non-singular plane curve of the form:

$$
C: y^{2}+h(x) y=f(x),
$$

where $h \in K[x]$ is of degree at most $g$ and $f \in K[x]$ is monic of degree $2 g+1$.

Note that, if $\operatorname{char}(K) \neq 2$, a change of variables can be performed to realize the curve in the form of $y^{2}=f(x)$ for $f \in K[x]$ monic of degree $2 g+1$. 
There are two natural ways to define amicable pairs in this context. One is to consider points on the curve $C$. In this case, we want two primes $p$ and $q$ of good reduction so that

$$
\# C\left(\mathbb{F}_{q}\right)=p \quad \text { and } \quad \# C\left(\mathbb{F}_{p}\right)=q .
$$

Such pairs do exist. The difficulty is that, for genus $g>1$, the set of points $C\left(\mathbb{F}_{p}\right)$ does not form a group, so one loses many of the tools used by Silverman and Stange to study amicable pairs.

Example 5.2. Let $C$ be the genus 2 hyperelliptic curve given by

$$
y^{2}=x^{5}+2 x^{4}+x^{2}+x+7 .
$$

Then, we have the following pairs of primes $(p, q)$ for $2 \leq p<q \leq 1000$ that satisfy $\# C\left(\mathbb{F}_{p}\right)=q$ and $\# C\left(\mathbb{F}_{q}\right)=p$ :

$$
\begin{array}{ccc}
(2,3), & (37,41), \quad(311,331), \quad(353,401), \\
(631,661), & (673,677), \quad(733,743), \quad(881,919) .
\end{array}
$$

Example 5.3. Let $C$ be the genus 2 hyperelliptic curve given by $y^{2}=x^{5}+x+1$. Then, we have the following pairs of primes $(p, q)$ for $2 \leq p<q \leq 1000$ that satisfy $\# C\left(\mathbb{F}_{p}\right)=q$ and $\# C\left(\mathbb{F}_{q}\right)=p$ :

$$
\begin{array}{cccc}
(41,47), \quad(83,109), \quad(97,107), & (139,151), & (263,293), \\
(359,383), & (421,457), & (431,463), & (523,557), \\
(733,769), & (743,757), & (911,937), & (977,983) .
\end{array}
$$

In future work, we plan to investigate the number of such pairs for genus 2 hyperelliptic curves and determine if one can obtain conjectural asymptotic formulas analogous to those given by Silverman and Stange.

The second method is to consider the Jacobian of the curve $C$. Every hyperelliptic curve has an associated geometric object called its Jacobian, denoted by $\mathrm{Jac}_{C}$. The Jacobian is a group, so we have some hope of using similar techniques to Silverman and Stange here.

The following is the statement of Hasse's theorem for hyperelliptic curves. Elliptic curves have genus 1 . Note that the next corollary gives the Hasse interval when $g=1$. 
Corollary 5.4. Let $C / \mathbb{F}_{p}$ be a hyperelliptic curve of genus $g$. Then,

$$
\left(p^{n / 2}-1\right)^{2 g} \leq \# \operatorname{Jac}_{C}\left(\mathbb{F}_{p^{n}}\right) \leq\left(p^{n / 2}+1\right)^{2 g} .
$$

Remark 5.5. For elliptic curves, the Jacobian $\operatorname{Jac}_{E}(K)$ is isomorphic to the group defined on the set $E(K)$. Thus, both potential generalizations specialize to the correct notion in the case of elliptic curves.

Theorem 5.6. Let $C$ be a hyperelliptic curve of genus $g>1$ defined over $\mathbb{Q}$ with good reduction at primes $p$ and $q$. Then, the statements

$$
\# \operatorname{Jac}_{C}\left(\mathbb{F}_{p}\right)=q \quad \text { and } \quad \# \operatorname{Jac}_{C}\left(\mathbb{F}_{q}\right)=p
$$

can only occur in the following cases:

(i) $g=2:(p, q)=(2,3)$ or $(p, q)=(3,5)$,

(ii) $g \geq 3:(p, q)=(2,3)$.

Proof. Suppose that we have such a pair $p$ and $q$. We apply the Hasse bound given above, with $n=1$, to obtain equations

$$
(\sqrt{q}-1)^{g} \leq \sqrt{p} \leq(\sqrt{q}+1)^{g}
$$

and

$$
(\sqrt{p}-1)^{g} \leq \sqrt{q} \leq(\sqrt{p}+1)^{g} .
$$

From this, we obtain

$$
(\sqrt{p}-1)^{g}-1 \leq \sqrt{q}-1 .
$$

It follows that we have

$$
\left((\sqrt{p}-1)^{g}-1\right)^{g} \leq(\sqrt{q}-1)^{g} \leq \sqrt{p} .
$$

Consider the polynomial

$$
f_{g}(x)=\left((x-1)^{g}-1\right)^{g}-x .
$$

Our above inequality implies that the only $p$ for which $p$ can be part of the pair $(p, q)$ occurs when $f_{g}(\sqrt{p}) \leq 0$. It easily follows that $f_{g}(x) \geq 0$ for all $x \geq 2.62$ and $g \geq 2$. Thus, we have that $p$ must be less than 7 . Since the same argument works for $q$, we have reduced to the possibilities that $p, q \in\{2,3,5\}$. Now, it is a simple case of plugging in these primes to determine which work. 


\section{REFERENCES}

1. Wieb Bosma, John Cannon and Catherine Playoust, The Magma algebra system I, The user language, J. Symb. Comp. 24 (1997), 235-265.

2. P. Mihăilescu, Primary cyclotomic units and a proof of Catalan's conjecture, J. reine angew. Math. 572 (2004), 167-195.

3. R. Schoof, Nonsingular plane cubic curves over finite fields, J. Comb. Theor. 46 (1987), 183-211.

4. J. Silverman and K. Stange, Amicable pairs and aliquot cycles for elliptic curves, Experimental Math. 20 (2011), 329-357.

5. W.A. Stein, et al., Sage mathematics software (Version 5.10), The Sage Development Team, 2013, http://www.sagemath.org.

Department of Mathematical Sciences, Clemson University, Clemson, SC 29634

Email address: jimlb@g.clemson.edu

Department of Mathematics, The College of William and Mary, WilliamsBURG, VA 23187

Email address: dheras@email.wm.edu

Department of Mathematical Sciences, Clemson University, Clemson, SC 29634

Email address: kevja@clemson.edu

Department of Mathematics and Statistics, East Tennessee State UniverSiTy, Johnson City, TN 37604

Email address: keatonr@etsu.edu

Department of Mathematics, University of California-Berkeley, BerkeLEY, CA 94720

Email address: trigalg693@berkeley.edu 